\title{
Data Adaptive Spectral Analysis of Unsteady Leakage Flow in an Axial Turbine
}

\author{
Konstantinos G. Barmpalias, ${ }^{1}$ Ndaona Chokani, ${ }^{1}$ Anestis I. Kalfas, ${ }^{2}$ and Reza S. Abhari ${ }^{1}$ \\ ${ }^{1}$ Laboratory for Energy Conversion (LEC), Swiss Federal Institute of Technology, ETH Zurich, 8092 Zurich, Switzerland \\ ${ }^{2}$ Department of Mechanical Engineering, Aristotle University of Thessaloniki, GR-54124 Thessaloniki, Greece
}

Correspondence should be addressed to Konstantinos G. Barmpalias, konstantinos.barmpalias@power.alstom.com

Received 5 October 2011; Accepted 13 February 2012

Academic Editor: David Japikse

Copyright ( $\odot 2012$ Konstantinos G. Barmpalias et al. This is an open access article distributed under the Creative Commons Attribution License, which permits unrestricted use, distribution, and reproduction in any medium, provided the original work is properly cited.

A data adaptive spectral analysis method is applied to characterize the unsteady loss generation in the leakage flow of an axial turbine. Unlike conventional spectral analysis, this method adapts a model dataset to the actual data. The method is illustrated from the analysis of the unsteady wall pressures in the labyrinth seal of an axial turbine. Spectra from the method are shown to be in good agreement with conventional spectral estimates. Furthermore, the spectra using the method are obtained with data records that are 16 times shorter than for conventional spectral analysis, indicating that the unsteady processes in turbomachines can be studied with substantially shorter measurement schedules than is presently the norm.

\section{Introduction}

The flows in turbomachines are inherently unsteady as it is only possible to have work exchange in the presence of a time-varying pressure field, as shown by Dean [1]:

$$
\frac{D h_{t}}{D t}=\frac{1}{\rho} \frac{\partial p}{\partial t}
$$

The unsteadiness within turbines arises due to the relative movement of the rotor and stator blade rows. Turbulence and stochastic processes give rise to random unsteadiness, whereas periodic unsteady phenomena, including waketo-blade interactions, potential field interactions, vortex interactions, and cavity flow interactions occur at the blade passing frequency or its harmonics. Langston [2] and Sieverding [3] referred extensively to secondary flows occurring in turbomachinery and relating them to losses. Mansour et al. [4] verified these loss generation mechanisms after having performed unsteady entropy measurements with the use of his fast response entropy probe in the harsh environment of the turbomachines. The loss mechanisms of these unsteady flow interactions are related to the efficiency of the turbomachinery. It is thus evident that an improved knowledge of the unsteady flow interactions can shed light on novel approaches to improve the efficiency of turbomachinery, as shown by Denton [5].

Making use of unsteady interactions can lead to potential loss generation reduction. Unsteady interactions of rotor inlet cavity and main flows on shrouded turbines can mitigate the adverse effects of the rotor tip passage vortex at its onset [6]. Blade-row interactions and especially the effect of wakes were exploited to control loss generation in ultrahigh-lift low pressure turbines [7]. Mathematical modeling of unsteadiness in turbomachinery flows can be used in order to improve design as well as producing integrated blading and flow path design. Chaluvadi et al. [8] developed a model to quantify vortex transport inside the downstream bladerow breaking down the unsteady loss generated upstream. Behr et al. [9] showed that clocking may impact on loss generation through axial and radial redistribution of low energy fluid while Barmpalias et al. [10] associated the stretching and tilting of the vortex with secondary loss generation.

Spectral analysis is a tool that can be used to quantify unsteady signals. Ullum et al. [11] reported on the onset and identification of the frequency of rotating stall based on the power spectrum analysis of velocity and pressure data in a centrifugal pump. In a completely different area, Chen Li and 

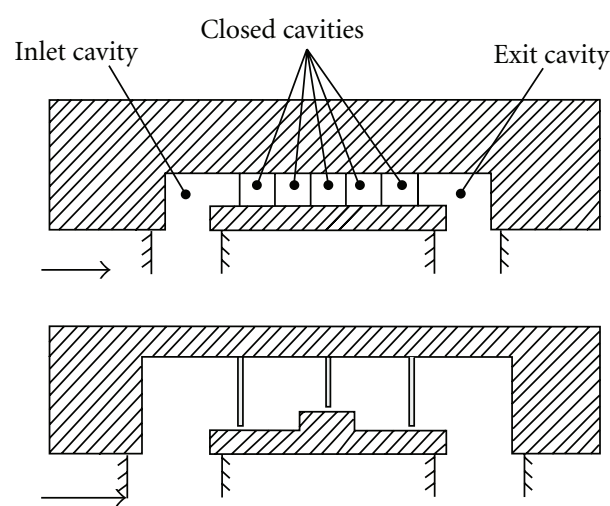

Figure 1: Examples of rotor tip labyrinth seal configurations (from Pfau [14]).

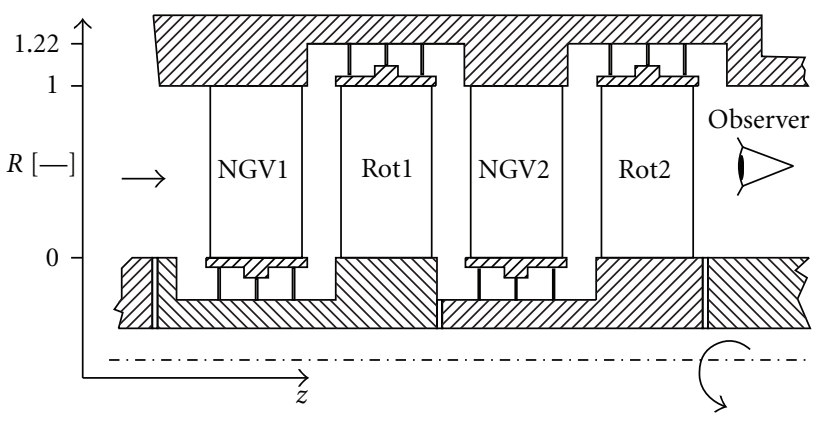

FIGURE 2: Meridional cut of the test section.

Li Ti-Pei [12] studied quasiperiodic oscillations in the X-ray emissions from a star using power spectrum density derived from an autoregressive model instead of the classical Fourier transformation so as to overcome the shortcomings of the latter. The work presented here is focused on the cavity flows that are associated with the rotor tip labyrinth seal, as seen in Figure 1. The rotor tip gap is required to provide a relative motion between the rotor blade and the casing. However, the associated unsteady cavity flow has a profound effect on the efficiency of the turbine. Pfau et al. [13] made highly resolved, three-dimensional flow measurements of the inlet cavity of a turbine rotor tip labyrinth seal in the two-stage axial research turbine. Additional measurements showed that this labyrinth seal configuration results in a turbine efficiency reduction of about $1.6 \%$. Pfau [14] showed that the gap size has a major effect on turbine losses. Unsteady wall pressure measurements, as shown in Figure 2, comparing 0.3\% and $1.0 \%$ gap cases indicate that there is a pronounced change in the cavity pressure distribution.

However, the changes in the pressure distribution could not be clearly discerned from the spectra. This difficulty arises due to the ensemble averaging procedures that are needed to improve the signal-to-noise ratio in Fourier-based power spectra. The objective of the current work is to demonstrate an alternative approach to obtaining spectral estimates. This alternate approach, the maximum entropy method of spectral analysis, has superior resolving power compared with more conventional spectral analysis.
TABLE 1: Main characteristics of the test turbine.

\begin{tabular}{lccc}
\hline $\begin{array}{l}\text { Pressure ratio } \\
\text { Max. power }\end{array}$ & $\begin{array}{c}1.32 \\
400 \mathrm{~kW}\end{array}$ & $\begin{array}{c}\text { Mass flow } \\
\text { Turbine speed }\end{array}$ & $\begin{array}{c}9.86 \mathrm{~kg} / \mathrm{sec} \\
2700 \mathrm{rpm}\end{array}$ \\
$\begin{array}{l}T_{\text {inlet }} \\
\text { Mach }\end{array}$ & $0.1-0.4$ & $\begin{array}{c}\text { Reynolds } \\
\text { number }\end{array}$ & $10^{5}$ \\
$\begin{array}{l}\text { Number of } \\
\text { blades on } \\
\text { rotor/stator } \\
\text { Blade passing } \\
\text { frequency }\end{array}$ & 42 & Tip diameter & $800 \mathrm{~mm}$ \\
\hline
\end{tabular}

The maximum entropy method (MEM) was first introduced by Burg [15], with his interest being focused on the analysis of geophysical data series. This method was subsequently used in related studies $[16,17]$. Burg commented on the better resolution and the more realistic power estimates of the MEM compared to the conventional methods of spectral estimation. Later Ulrych and Clayton [18] reviewed the principles of maximum entropy spectral analysis and the closely related topic of autoregressive time series modeling. Barrodale and Erickson $[19,20]$ developed an algorithm to solve the underlying least-squares linear prediction problem in maximum entropy spectral analysis. At the same time, Theodoridis and Cooper [21] applied the maximum entropy spectral analysis technique to signals with spectral peaks of finite width and compared their results to that of the conventional Fourier method. They reported that the spectra were smoother and better resolved. Well-defined spectral peaks could be obtained using fewer points. Maximum entropy methods for spectral estimates now have broader acceptance and use in the field of vibration data analysis [22] and even in the textile industry [13]. Morgenstern and Chokani [23] applied MEM for purposes of spectral analysis of pressure signals in hypersonic flows past open cavities and noted that extremely short data lengths are required to yield the spectra.

In general, the method is suited to data with poor signalto-noise ratios and also for extremely short data lengths. In the next section we briefly review conventional methods of spectral analysis and highlight their shortcomings. The formulation of the maximum entropy method of spectral analysis is then outlined, and the results of its application to the unsteady wall pressure measurements of Pfau [14] are presented. Finally, we conclude with a summary of the work.

1.1. The Research Facility. The measurements were performed in the "LISA" two-stage axial research turbine at the Laboratory for Energy Conversion (LEC), ETH Zurich. The turbine inlet temperature is kept constant at $400 \mathrm{~K}$ with an accuracy of $0.9 \mathrm{~K}$. A DC generator maintains a constant operating speed of $2700 \pm 0.5 \mathrm{RPM}( \pm 0.02 \%)$. A more detailed description of the test facility is available in Schlienger et al. [24]. The main characteristics of the turbine are summarized in Table 1. 


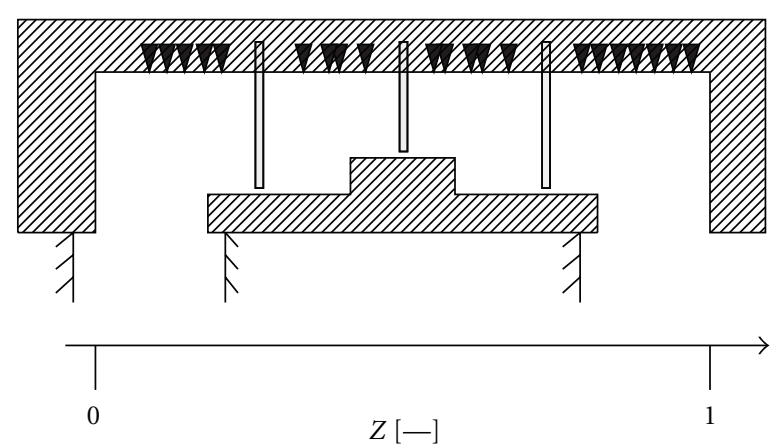

(a)

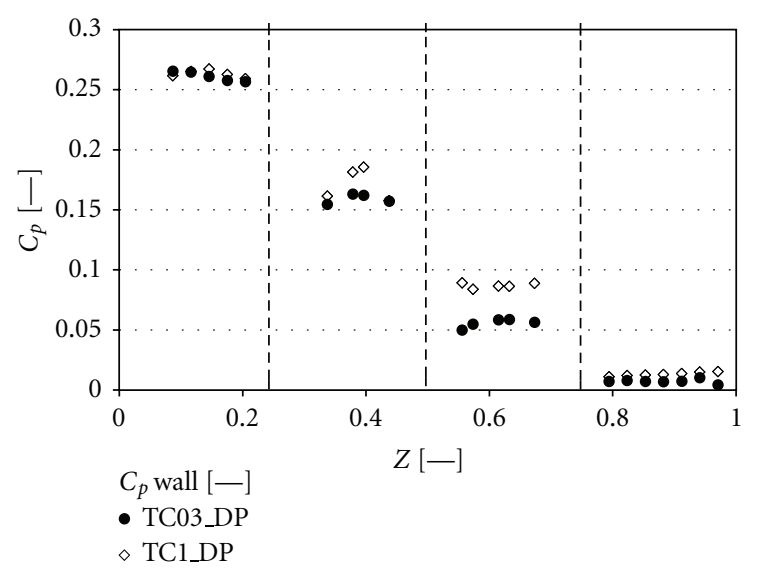

(b)

Figure 3: Axial locations of pressure taps in labyrinth groove (a) and pitch-wise averaged wall pressures $C p$ for $0.3 \%$ gap case (TC03_DP) and 1\% gap case (TC1_DP) (b) (from Pfau [14]).

The constant annulus of the turbine and the four blade rows are depicted in Figure 2. The geometry under investigation is representative of a steam turbine. The unsteady wall measurements performed during this campaign were made at the rotor inlet cavity downstream of the 2 nd stator.

1.2. Unsteady Wall Pressure Measurements. The unsteady pressure measurements presented here were acquired at the inlet cavity of the 2nd rotor shown in Figure 3. Six pressure holes in an axial direction, distanced $2.5 \mathrm{~mm}$ apart, were used. The inlet cavity itself has a $25 \mathrm{~mm}$ axial wall. The 1 st measurement hole is located $15 \%$ downstream from the upstream radial wall. The potential to circumferentially clock the cavity ring against the stator permits multimeasurements in the circumferential direction. One stator pitch is thus covered by 23 circumferential measurements.

\section{Conventional Spectral Analysis}

The most widely used method of spectral estimation is that based on the use of the fast Fourier transformation. In this approach, if we consider a uniformly sampled time series $x_{n}$ where $n=1,2, \ldots, N$, then the estimate of the power spectral density is given as

$$
\operatorname{PSD}_{m}=\frac{1}{N \cdot \Delta t}\left(X_{m} X_{m}^{*}\right) .
$$

The index $m$ denotes the discrete frequency, $f_{m}$, at which the power spectral density is estimated. The FFT approach for spectral estimates is widely used as computationally efficient methods for the determination of (2) have been developed. However, although the FFT technique is widely used, there are several aspects of the technique that limit its reliability. Firstly, the technique assumes that the signal of the sampled time series is a linear series of sine waves. Secondly, the spectral resolution is inversely proportional to the duration of the signal; therefore, for small frequency resolution a long signal is required. Thirdly, the signal is assumed to be stationary-that is the processes generating the signal are assumed to be the same from the beginning through to the end of the analysis period. Furthermore, a window function is required to minimize the leakage in the spectral estimates; this window function distorts the estimates in adjacent frequencies. Finally, when the signal-to-noise ratio of the signal is large, the spectral estimates are unreliable. Alternatively, the Wavelet Transform (WT) may be employed so as to overcome the FFT shortcomings. This would allow for the analysis of signals that have highly concentrated time localized high-frequency components superimposed on longer lived low-frequency components. This feature would allow for feature detection apart from signal noise removal and data compression [25]. The WT has a time window interval width that is inversely proportional to its wave width for all frequencies.

\section{Data Adaptive Spectral Analysis}

In an attempt to overcome the aforementioned limitations of FFT conventional spectral analysis method, Burg [15] suggested the maximum entropy method for spectral analysis. Burg's interest was in the analysis of geophysical data series, and in that application it was found that this new method gave better spectral resolution and more realistic estimates of power. The development of the MEM as suggested by Burg is as follows. The entropy of a Gaussian process is given as:

$$
H=\frac{1}{4 f_{N}} \int_{-f_{N}}^{f_{N}} \log \operatorname{PSD}(f) d f .
$$

In terms of the auto covariance of the data time series, (3) can be rewritten as

$$
H=\frac{1}{4 f_{N}} \int_{-f_{N}}^{f_{N}} \log \left\lfloor\sum_{k=-\infty}^{\infty} \rho(k) e^{-2 \pi i f_{k} \Delta t} d f\right\rfloor .
$$

If $H$ is maximized by the use of Lagrange multipliers, subject to the constraint that the spectral estimate is consistent with the known autocovariances, $\rho(k),-p<k<p$, the solution to the variational problem is

$$
P_{\mathrm{MEM}}(f)=\frac{P_{p} \Delta t}{\left|1-\sum_{k=1}^{p} a_{k} e^{-2 \pi i f_{k} \Delta t}\right|^{2}} .
$$




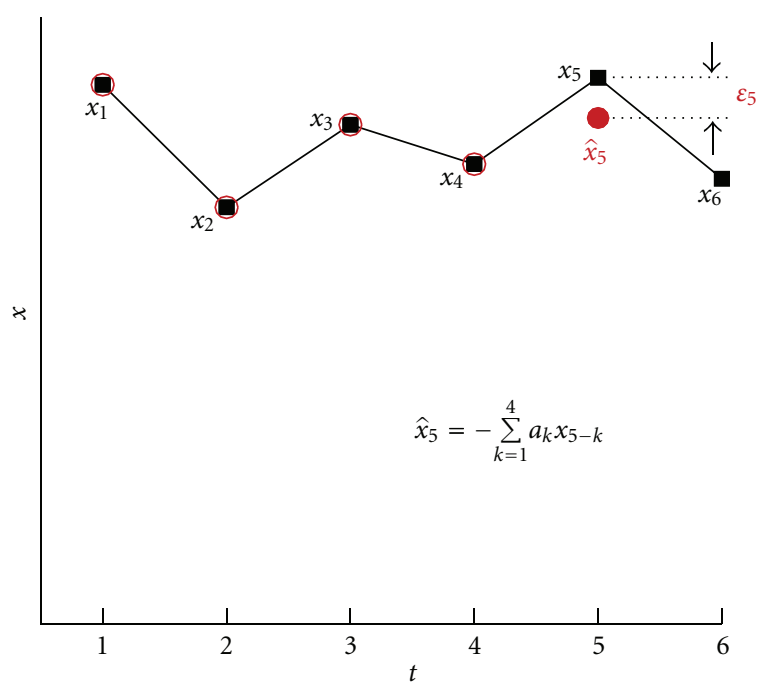

FIgUre 4: The Maximum entropy method of spectral analysis from the perspective of time series modeling. In this example, black squares denote measured data; unfilled red circles show past measured values that are used to estimate a modeled data point (filled red symbol).

Equation (5) is the expression for the spectral estimates obtained from MEM and is equivalent to the estimate derived using the FFT method, shown by (2). The maximizing of (4) results in the rather unfortunately named-in the sense that it is confusing from the perspective of examining loss generation in turbomachines_maximum entropy method for spectral analysis.

Van den Bos [26] investigated the duality between the maximum entropy method for spectral analysis and autoregressive representation. If we briefly review this duality, the interpretation of (5) is then clearer. Consider an autoregressive model that is consistent with the measured data:

$$
\hat{x}_{n}=-\sum_{k=1}^{p} a_{k} x_{n-k} .
$$

Note that (6) represents a pth-order model, in which the modeled data $\hat{x}_{\mathrm{n}}$ are determined from a finite number, $p$, of previous measured data points.

Thus, for example, if we have a 4 th order model, a linear combination of 4 past values is used to predict each modeled data point, as in Figure 4. Although we lose data points, in the sense that if $N$ measured data points are available, then only $(N-p)$ modeled data points can be determined, and the modeled data are solely based on known, measured data. The total squared error between the modeled and measured data is given as

$$
\begin{aligned}
E & =\sum_{n=p}^{N-1}\left(\hat{x}_{n}-x_{n}\right)^{2} \\
& =\sum_{n=p}^{N-1}\left(-\sum_{k-1}^{p} a_{k} x_{n-k}-x_{n}\right)^{2} .
\end{aligned}
$$

The minimization of (7) with respect to the coefficients $a_{1}$, $a_{2}, \ldots, a_{p}$ then yields a spectrum of $(N-p)$-length time series of error, $\varepsilon_{n}=\hat{x}_{n}-x_{n}$, that is equivalent to that of "white" noise. It is therefore evident from (3) that the uncertainty $\varepsilon_{n}$ is maximized, and therefore this maximization yields the most random (i.e., maximum entropy) error between the modeled and measured data. On the other hand, for this maximization (therefore known coefficients $a_{1}, a_{2}, \ldots, a_{p}$ ) (6) represents a time series model that is adapted to the measured data. Thus we prefer to refer to Burg's approach as a data adaptive spectral analysis method.

The basic working equation for the spectral estimates determined using the data adaptive spectral analysis method is given by (5). Barmpalias et al. [10] present an algorithm to determine the coefficients $a_{1}, a_{2}, \ldots, a_{p}$ and the model constant, $P_{p}=P_{p-1}\left(1-a_{p}^{2}\right)$, from the measured data.

It is pertinent to highlight two salient differences between the spectral estimates from conventional spectral analysis, as shown in (2), and from data adaptive spectral analysis, as in (5). Firstly in (5) no windowing is used, unlike in (2). Thus (5) is based on actual measured data, whereas (2) is based on measured data that are modified by the windowing procedure. Secondly, in (5) the frequency of the spectral estimate, $f$, is an independent variable. Thus the spectral resolution in (5) is independent of the sampling frequency and the user chooses the desired spectral resolution. By contrast, in conventional spectral analysis the frequency resolution is the inverse of the duration of the length of the measured data, and therefore in (2) high frequency resolution can only be accomplished by having a long measured signal. Burg [15] has noted that (5) is well suited for short data lengths.

The determination of the order, $p$, in the time series model, (6), is of central importance to the data adaptive spectral analysis method. Stated simply, if $p$ is too small, then a smooth spectrum is obtained; on the other hand, if $p$ is too large, then there are spurious peaks in the spectrum. The most commonly used approaches to determine the optimum $p$ involve the final prediction error, Akaike [25]. That is given by

$$
\operatorname{FPE}_{p}=\frac{N+p+1}{N-p-1} P_{p}
$$

and/or the Akaike [22] information criterion, that is,

$$
\mathrm{AIC}_{p}=N \operatorname{Nog}_{10} P_{p}+2 p .
$$

The optimum $p$ is determined as the first occurrence of $\mathrm{FPE}_{p+1}>\mathrm{FPE}_{p}$ for successively increasing $p$. This criterion was not found to be reliable in the present work. Instead, as the primary focus in this work is periodic unsteady phenomena and cavity flow interactions that occur at the blade passing frequency, the optimum $p$ is determined as that value for which the FPE first exhibits a local maximum in the region of interest as $p$ is increased. This range is bounded by the occurrence of a smooth spectrum for relatively small $p$ and by spurious peaks in the spectrum when a certain value of $p$ is exceeded. For this application the optimum $p$ was found to be within the range of 200 to 400 . As seen in 


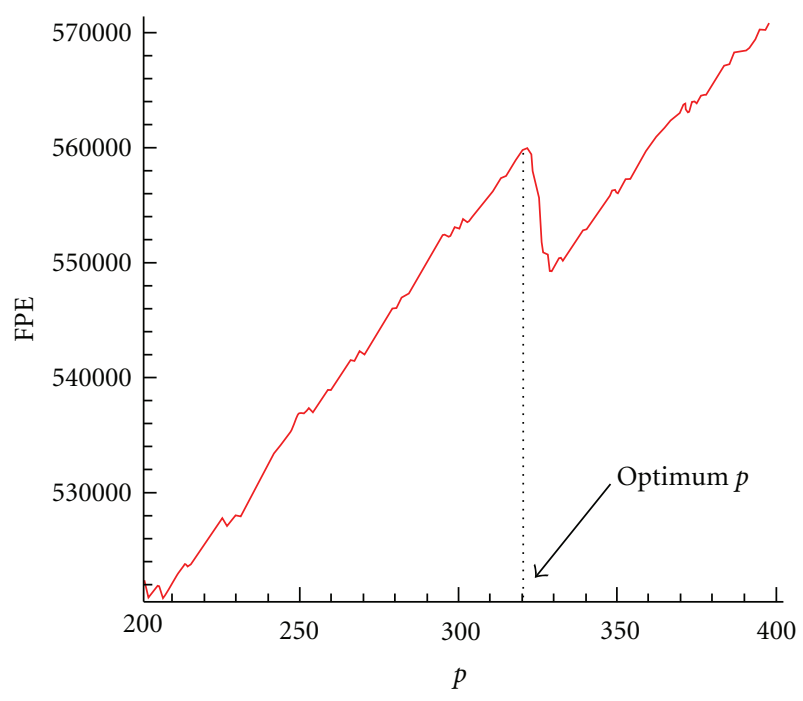

FIGURE 5: Determining the optimum $p$ as the first occurrence of a local maximum in FPE as $p$ increases.

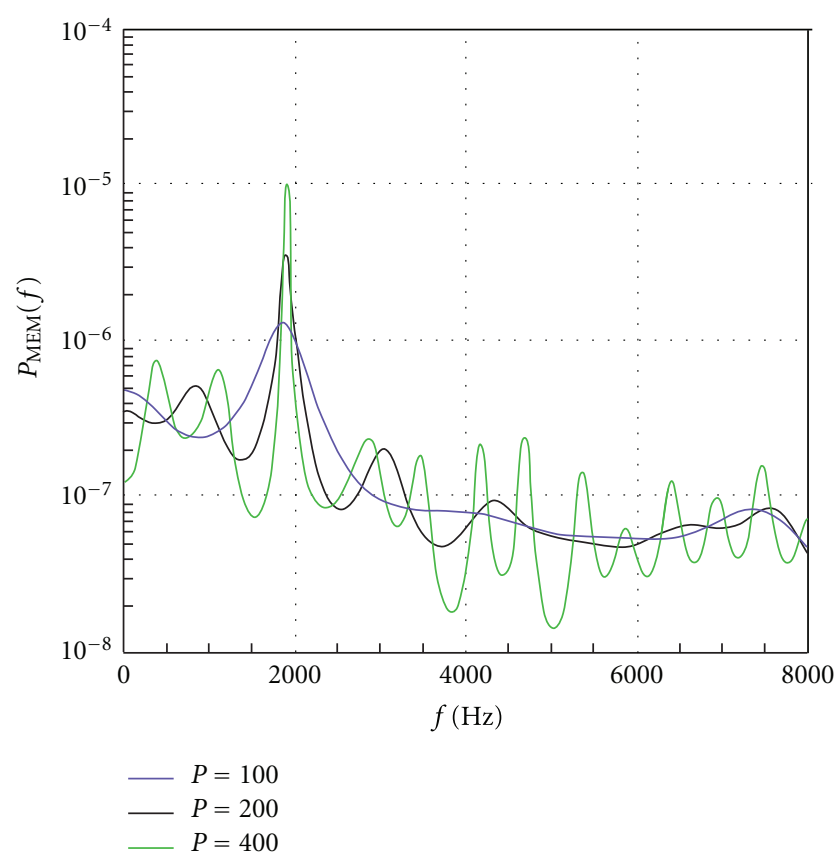

Figure 6: The effect of the time series model order, $p$, see (6), on the spectra predicted using the data adaptive spectral analysis method.

Figure 5, FPE has a local maximum at $p=320$, which is then taken as the optimum $p$.

\section{Results and Discussion}

An analysis of the unsteady wall pressure measurements of Pfau [14] is presented to illustrate the data adaptive spectral analysis method. The labyrinth seal configuration consists of 4 cavities with a gap size of $0.1 \mathrm{~mm}$, which corresponds to $1 \%$ of blade height. The data are sampled at a rate of $200 \mathrm{kHz}$.

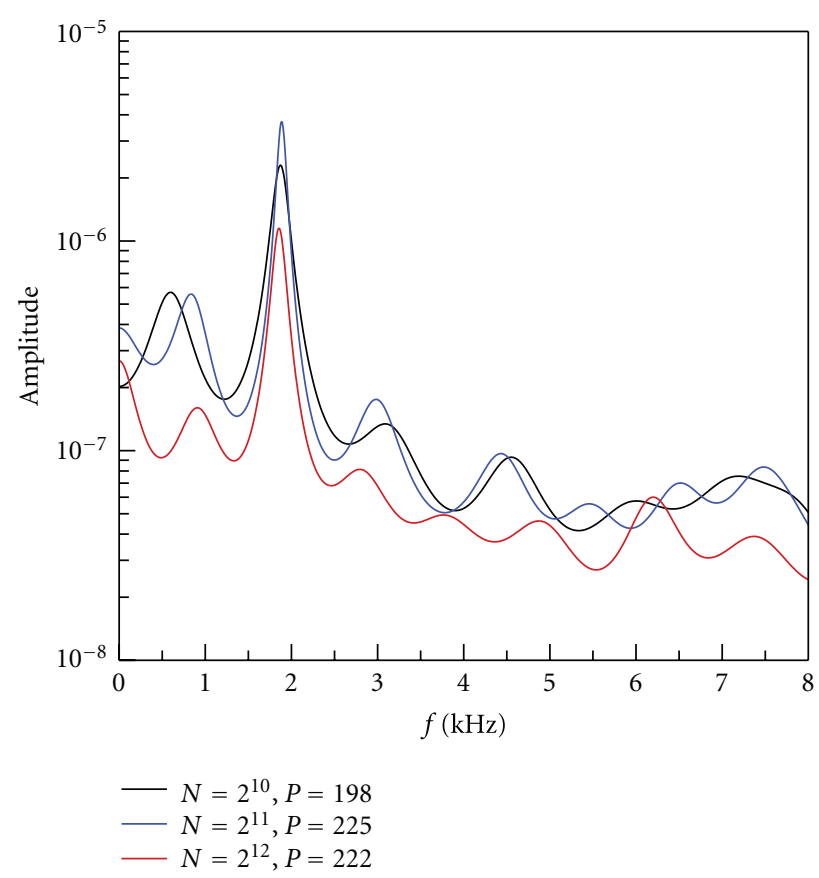

Figure 7: The effect of the length of measured data, $N$, on the spectra predicted using the data adaptive spectral analysis method.

For the present work the data acquired in cavity 1 at the midcircumference station are presented.

Figure 6 examines the effect of the time series model order, $p$, as shown in (6) on the spectra predicted using the data adaptive spectral analysis method. Three different model orders, 100, 200, and 400, are examined. For all three cases, the spectral resolution is $12.2 \mathrm{~Hz}$ and the spectra show a dominant peak at the blade passing frequency, $1840 \mathrm{~Hz}$. The optimum value of $p$ is 200 , and the corresponding spectrum shows additional peaks at the harmonic frequency and nonlinear interaction frequencies (i.e., subharmonic frequency and difference interaction (fundamental and subharmonic) frequency). For $p=100(<200)$, it can be seen that the spectrum is relatively smooth compared to the spectrum of the optimum $p$, where, for example, the harmonic frequency is not detected. For $p=400$ ( $>200$ ), on the other hand, there are many spurious peaks in the spectrum. These results clarify the importance of determining the order of the time series model in the data adaptive spectral analysis method.

The effect of the length of the measured data record, $N$, on the spectra predicted using the data adaptive spectral analysis method is examined in Figure 7. Three different data record lengths, $2^{10}, 2^{11}$, and $2^{12}$, are examined. For each data record length the spectral resolution is specified as $12.2 \mathrm{~Hz}$, and the optimum order of time series model, $p$, is determined and found to be 198, 225, and 222, respectively, for the three data record lengths. It can be seen that for all three cases, the spectra show essentially the same information. This result shows the ability of the data adaptive spectral analysis method to extract the same spectral information for both long and short data records. It should be noted that there are small differences in the spectral 


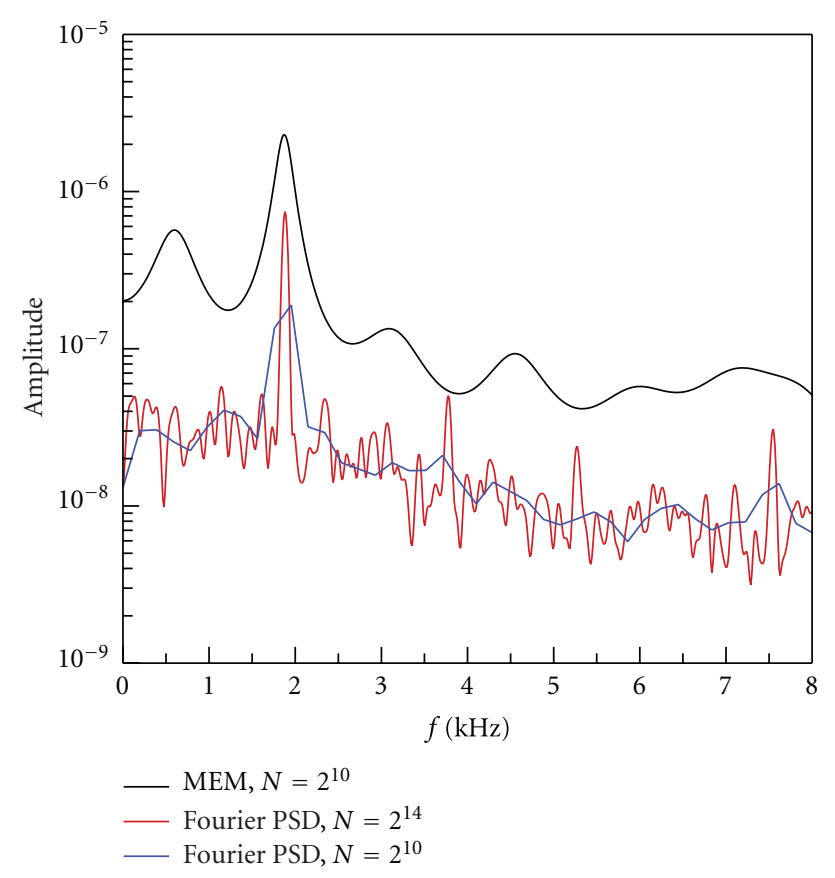

FIgURE 8: Comparison of data adaptive and conventional spectral estimates of power spectral densities.

amplitudes. These differences arise since the model constant, $P_{p}=P_{p-1}\left(1-a_{p}^{2}\right)$, in (5) is related to the coefficients of the time series model. However, the normalized spectra are indistinguishable, as noted by Burg [15].

Figure 8 compares the power spectral estimates of the data adaptive and conventional methods of spectral analysis. For sake of clarity, the conventional spectral estimates are offset by one decade from the data adaptive spectral estimate. In all three cases, the peak at the blade passing frequency is identified as the most dominant feature of the spectra. In the case where the data record lengths are the same, $N=2^{10}$, it is evident that the conventional spectral estimate has a relatively poor spectral resolution, 195.2 Hz. Furthermore, in the conventional spectral estimate, no peaks other than that at the blade passing frequency are clearly identified. In order to improve the spectral resolution in the conventional spectral estimate to the spectral resolution, $12.2 \mathrm{~Hz}$, of the data adaptive spectral estimate, the data record length must be increased by a factor 16 to $N=2^{14}$. The resultant spectrum, as seen in Figure 8, captures additional peaks in the spectra. However, it is evident that the conventional spectral estimate is noisier. On the other hand, the data adaptive spectral estimate, which is obtained with a shorter data record, yields a smoother spectrum. It is therefore evident that using the data adaptive spectral analysis method, the measurement schedule could be reduced by up to factor 16 in terms of data acquisition, while still being able to extract available information from the spectra.

\section{Concluding Remarks}

A data adaptive spectral analysis method, which has previously been used in the analysis of geophysical data series, has been introduced in the context of examining the periodic unsteadiness within turbines. This data adaptive spectral analysis method employs a time series model of measured data. The spectrum of the model's error is the most random (i.e., maximum entropy) that is consistent with measured data. No windows are employed in the determination of the spectral estimates. Spectral estimates are obtained with a data record that is 16 times shorter than that required for Fourier power spectrum estimates. The use of shorter data records has the potential to reduce measurement schedules for measurement test campaigns.

The application of this data adaptive spectral analysis method (maximum entropy method) as employed within the context of this study can be summarized in the following steps for a given number of data points, $N$.

(1) Calculate the final prediction error, FPE, using (8) as the order of $p$ is increased.

(2) Determine the optimum order of $p$ as the first occurrence of a maximum in FPE, as in Figure 5.

(3) Calculate the spectrum using (5).

\section{Nomenclature}

$a_{k}: \quad$ Coefficients of time series model

$\varepsilon_{n}: \quad$ Model error, $\hat{x}_{n}-x_{n}$

E: $\quad$ Total error

F: $\quad$ Frequency

$f_{\mathrm{bp}}: \quad$ Blade passing frequency

$f_{m}: \quad$ Discrete frequency, $(m-1) \Delta f$

$f_{N}: \quad$ Nyquist frequency

$\Delta f: \quad$ Spectral resolution

$H$ : Information entropy of a Gaussian process

$M$ : Index for spectral estimate

$N$ : $\quad$ Index for data time series

$P$ : $\quad$ Order of time series model, or pressure

$P_{p}: \quad$ Output power of $p$-length time series model

$P_{\text {MEM }}$ : MEM power spectral estimate

$R: \quad$ Blade span

T: $\quad$ Time

$\Delta t$ : Sampling rate

$x_{n}: \quad$ Measured data time series

$\hat{x}_{n}: \quad$ Modeled data time series

$X_{m}$ : Fourier transformation of $x_{n}$

$X_{m}^{*}: \quad$ Complex conjugate of $X_{m}$

$Z: \quad$ Axial direction

$T_{\text {inlet: }}$ : Temperature at stage inlet

$p_{\text {exit }}$ : Pressure at turbine exit.

\section{Abbreviations}

FFT: Fast Fourier transformation

FPE: Final prediction error 
MEM: Maximum entropy method for spectral analysis

$\mathrm{PSD}_{m}$ : Power spectral density estimate.

\section{Acknowledgment}

The authors are grateful to Axel S. Pfau for making available the unsteady wall pressure data used here.

\section{References}

[1] R. C. Dean, "On the necessity of unsteady flow in fluid machines," ASME Journal of Basic Engineering, vol. 81, pp. 2428, 1959.

[2] L. S. Langston, "Secondary flows in axial turbines-a review," Annals of the New York Academy of Sciences, vol. 934, pp. 1126, 2001.

[3] C. H. Sieverding, "Recent progress in the understanding of basic aspects of secondary flows in turbine blade passages," Journal of Engineering for Gas Turbines and Power, vol. 107, no. 2, pp. 248-257, 1985.

[4] M. Mansour, N. Chokani, A. I. Kalfas, and R. S. Abhari, "Time-resolved entropy measurements using a fast response entropy probe," Measurement Science and Technology, vol. 19, no. 11, Article ID 115401, 2008.

[5] J. D. Denton, "Loss mechanisms in turbomachines," Journal of Turbomachinery, vol. 115, no. 4, pp. 621-656, 1993.

[6] K. G. Barmpalias, A. I. Kalfas, and R. S. Abhari, "Design considerations for axial steam turbine rotor inlet cavity volume and length scale," in ASME Turbo Expo, Vancouver, Canada, June 2011.

[7] H. P. Hodson and R. J. Howell, "Bladerow interactions, transition, and high-lift aerofoils in low-pressure turbines," Annual Review of Fluid Mechanics, vol. 37, pp. 71-98, 2005.

[8] V. S. P. Chaluvadi, A. I. Kalfas, H. P. Hodson, H. Ohyama, and E. Watanabe, "Blade row interaction in a high-pressure steam turbine," Journal of Turbomachinery, vol. 125, no. 1, pp. 14-24, 2003.

[9] T. Behr, L. Porreca, T. Mokulys, A. I. Kalfas, and R. S. Abhari, "Multistage aspects and unsteady effects of stator and rotor clocking in an axial turbine with low aspect ratio blading," in ASME Turbo Expo, pp. 1211-1222, June 2004.

[10] K. G. Barmpalias, A. I. Kalfas, N. Chokani, and R. S. Abhari, "The dynamics of the vorticity field in a low solidity axial turbine," in ASME Turbo Expo, June 2008.

[11] U. Ullum, J. Wright, O. Dayi et al., "Prediction of rotating stall within an impeller of a centrifugal pump based on spectral analysis of pressure and velocity data," Journal of Physics: Conference Series, vol. 52, no. 1, pp. 36-45, 2006.

[12] L. Chen and T. P. Li, "Autoregressive spectral estimation for quasi-periodic oscillations," Chinese Journal of Astronomy and Astrophysics, vol. 5, no. 5, pp. 495-507, 2005.

[13] A. Pfau, J. Schlienger, D. Rusch, A. I. Kalfas, and R. S. Abhari, "Unsteady flow interactions within the inlet cavity of a turbine rotor tip labyrinth seal," Journal of Turbomachinery, vol. 127, no. 4, pp. 679-688, 2005.

[14] A. Pfau, Loss mechanisms in labyrinth seals of shrouded axial turbines ETH Dissertation No. 15226, Swiss Federal Institute of Technology, ETH, Zürich, Switzerland, 2003.

[15] J. P. Burg, Maximum entropy spectral analysis, Ph.D. thesis, Department of Geophysics, Stanford University, 1975.
[16] R. O. Vicente and R. G. Currie, "Maximum entropy spectrum of long-period polar motion," Royal Astrological Society Geophysical Journal, vol. 46, pp. 67-73, 1976.

[17] H. R. Radoski, E. J. Zawalick, and P. F. Fougere, "The superiority of maximum entropy power spectrum techniques applied to geomagnetic micropulsations," Physics of the Earth and Planetary Interiors, vol. 12, no. 2-3, pp. 208-216, 1976.

[18] T. J. Ulrych and R. W. Clayton, "Time series modelling and maximum entropy," Physics of the Earth and Planetary Interiors, vol. 12, no. 2-3, pp. 188-200, 1976.

[19] I. Barrodale and R. E. Erickson, "Algorithms for least-squares linear prediction and maximum entropy spectral analysispart I: theory," Geophysics, vol. 45, no. 3, pp. 420-432, 1980.

[20] I. Barrodale and R. E. Erickson, "Algorithms for least-squares linear prediction and maximum entropy spectral analysispart II: fortran program," Geophysics, vol. 45, no. 3, pp. 433$446,1980$.

[21] S. Theodoridis and D. C. Cooper, "Application of the maximum entropy spectrum analysis technique to signals with spectral peaks of finite width," Signal Processing, vol. 3, no. 2, pp. 109-122, 1981.

[22] T. M. Romberg, A. G. Cassar, and R. W. Harris, "A comparison of traditional Fourier and maximum entropy spectral methods for vibration analysis," Journal of Vibration, Acoustics, Stress, and Reliability in Design, vol. 106, no. 1, pp. 36-39, 1984.

[23] A. Morgenstern and N. Chokani, "Hypersonic flow past open cavities," AIAA Journal, vol. 32, no. 12, pp. 2387-2393, 1994.

[24] J. Schlienger, A. Pfau, A. I. Kalfas, and R. S. Abhari, "Effects of labyrinth seal variation on multistage axial turbine flow," in ASME Turbo Expo, pp. 173-185, June 2003.

[25] H. Akaike, "Fitting autoregressive models for prediction," Annals of the Institute of Statistical Mathematics, vol. 21, pp. 243247, 1969.

[26] A. van den Bos, "Alternative interpretation of maximum entropy spectral analysis," IEEE Transactions on Information Theory, vol. 17, no. 4, pp. 493-494, 1971. 



Submit your manuscripts at

http://www.hindawi.com
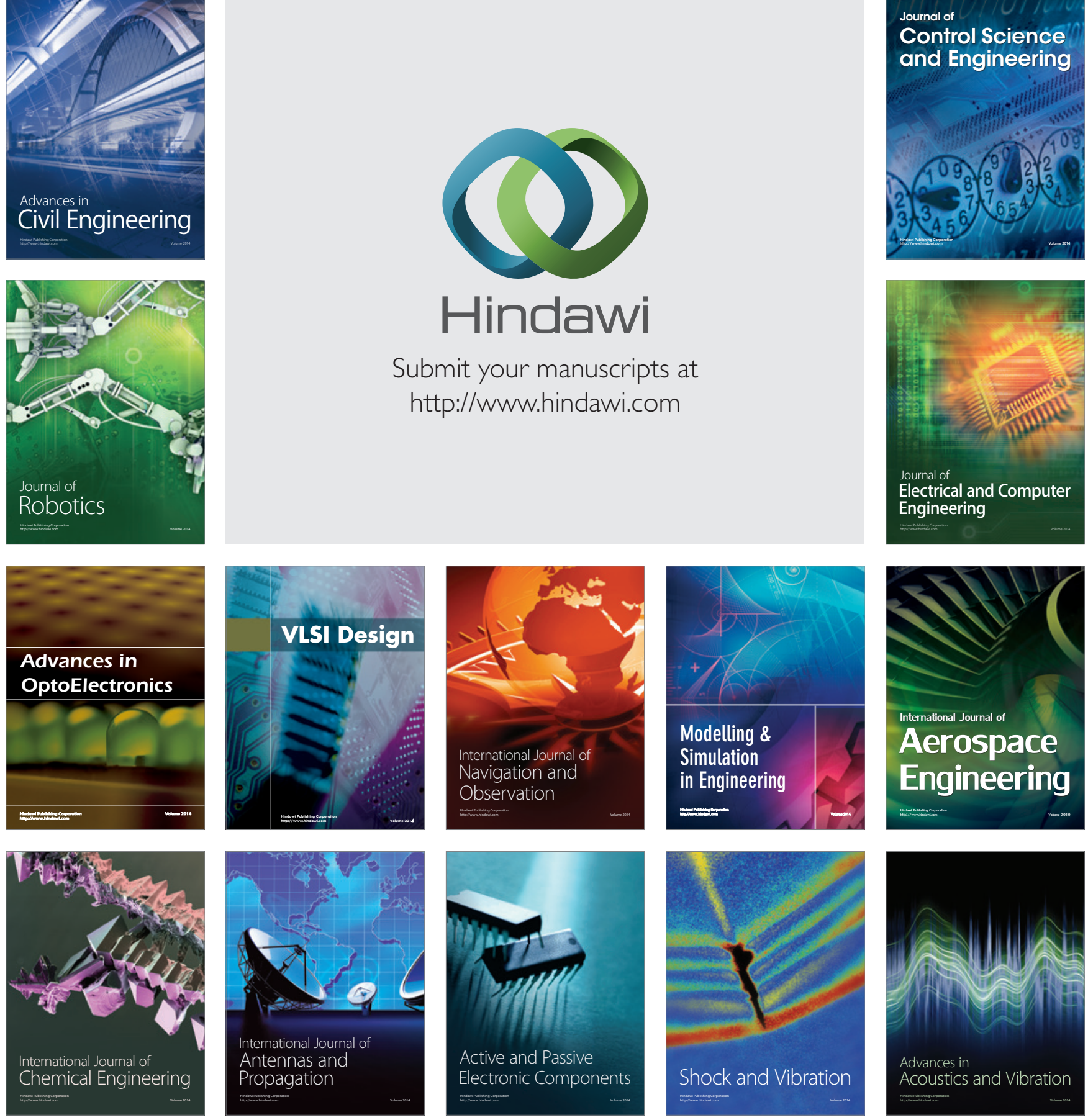